\title{
Measuring household food security in subsistence paddy farming sector in Sri Lanka: development of household food insecurity index (HFSI)
}

\author{
R. L. D. K. Malkanthi ${ }^{1}$, K. D. R. R. Silva ${ }^{1}$ and J. M. U. K. Jayasinghe ${ }^{2}$ \\ ${ }^{1}$ Department of Applied Nutrition, Faculty of Livestock, Fisheries and Nutrition, Wayamba University of Sri Lanka, \\ Makandura, Gonawila (60170), Sri Lanka and ${ }^{2}$ Department of Agribusiness Management, Faculty of Agriculture 7 \\ Plantation Management, Wayamba University of Sri Lanka, Makandura, Gonawila (60170), Sri Lanka
}

Food security is a complex and multidimensional phenomenon. As such, its measurement may entail and benefit from the combination of both 'qualitative-subjective' and 'quantitative-objective' indicators. Therefore, this study was aimed at developing and validating a composite household food insecurity index (HFSI) by taking into account three dimensions of food security i.e. (i) accessibility, (ii) availability (iii) utilisation to assess the level of household food insecurity in rural subsistence paddy farming sector in Sri Lanka where the poverty and malnutrition is high.

Study was conducted in two phases. In Phase I, case studies and pilot study were carried out to identify food security attributes and to verify same. Eighty subsistence paddy farming households from twenty Divisional Secretariats (DS) divisions in ten districts were studied to identify the indicators of food insecurity and to establish methodology. In the Phase II, a questionnaire based survey and assessing nutrition status by means of dietary and anthropometry were done with a sample of 300 subsistence paddy farming households from ten DS divisions in five districts in Sri Lanka. Seven indicators i.e. economic (ECO), social (SOC), dietary (DIET), nutrition (NUT), water and sanitation (WAS), perception on food consumption (PFC) and coping strategies (COP) were identified to develop the index. A composite additive index was developed by assigning weights for above identified seven indicators. The developed index was, HFSI $=0.18 * \mathrm{ECO}+0.19 * \mathrm{SOC}+0.11 *$ DIET $+0.08 \mathrm{NUT}+0.11 \mathrm{WAS}+0.08 * \mathrm{COP}+0.25 * \mathrm{PFC}$. The contribution from each indicator for the HFSI was $18,19,11,8,11,8$ and $25 \%$ for economic, social, dietary, nutrition, water and sanitation, perception on food consumption and coping strategies, respectively. Households were categorised into four categories based on the scores obtained for the index i.e. severe food insecure (if the score value is $\geq 75$ ), moderate food insecure (if the score value is $51-74$ ), less food insecure (if the score value is 26-50) and least food insecure (if the score value is $\leq 25$ ).

Prevalence of severe food insecurity, medium food insecurity, less food insecurity and least food insecurity were $9,57,27$ and $7 \%$, respectively. Sixty-six percent of the households had high food insecurity (severe and moderate together). High food insecure household had significantly $(P<0.05)$ lower net income per capita, energy per equivalent adult, household dietary diversity and $\mathrm{Hb}$ levels in children $<5$ years and higher WAZ (low-weight-for-age) in children $<5$ years, household perception on food consumption score and coping strategy score. Multiple logistic regression model showed that net income per capita, receiving government assistance, food ratio, level of education of father, level of education of mother, household energy adequacy, household dietary diversity, prevalence of anaemia among $<5$ years old children and access to safe drinking water in household were significantly associated with food insecurity score $\left(R^{2}=0.772 ; P<0.0001\right)$.

We developed a composite index to assess the level of household food insecurity in the rural subsistence paddy farming households in Sri Lanka. Based on the developed HFSI, the prevalence of severe food insecure, medium food insecure, less food insecure and least food insecure were 9, 57, 27 and 7\%, respectively in the rural subsistence paddy farming households in Sri Lanka. In conclusion, HFSI could be used to capture multidimensional phenomenon of household food insecurity in the rural subsistence paddy farming households in Sri Lanka. 